\title{
Phase III trial of chemotherapy using 5-fluorouracil and streptozotocin compared with interferon $\alpha$ for advanced carcinoid tumors: FNCLCC-FFCD 9710
}

\author{
Laetitia Dahan ${ }^{1 *}$, Frank Bonnetain ${ }^{2 *}$, Philippe Rougier ${ }^{3}$, Jean-Luc Raoul ${ }_{4}^{4}$, \\ Eric Gamelin ${ }^{5}$, Pierre-Luc Etienne ${ }^{6}$, Guillaume Cadiot ${ }^{7}$, Emmanuel Mitry ${ }^{4}$,' \\ ${ }_{\text {Denis Smith }}{ }^{8}$, Frédérique Cvitkovic ${ }^{9}$, Bruno Coudert ${ }^{10}$, Floriane Ricard ${ }^{1}$, \\ Laurent Bedenne ${ }^{1}$, Jean-François Seitz ${ }^{1}$ for the Fédération Francophone de \\ Cancérologie Digestive (FFCD) and the Digestive Tumors Group of the \\ Fédération Nationale des Centres de Lutte Contre le Cancer (FNCLCC)
}

\author{
${ }^{1}$ Assistance Publique, Hôpitaux de Marseille, Hôpital Timone, Université de la Méditerranée, CHU Timone, 264 rue Saint Pierre, \\ 13385 Marseille Cedex 5, France \\ ${ }^{2}$ FFCD, Dijon, France \\ ${ }^{3}$ AP-HP, Hôpital Ambroise Paré, Boulogne, France \\ ${ }^{4}$ Centre Eugène Marquis, Rennes, France \\ ${ }^{5}$ Centre Paul Papin, Angers, France \\ ${ }^{6}$ Clinique Armoricaine, Saint Brieuc, France \\ ${ }^{7}$ Hopital Robert Debré, Reims, France \\ ${ }^{8} \mathrm{CHU}$ Haut Leveque, Pessac, France \\ ${ }^{9}$ Centre René Huguenin, Saint Cloud, France \\ ${ }^{10}$ Centre François Leclerc, Dijon, France \\ (Correspondence should be addressed to L Dahan; Email: laetitia.dahan@mail.ap-hm.fr) \\ *(L Dahan and F Bonnetain contributed equally to this work)
}

\begin{abstract}
The aim of this randomized multicenter phase III trial was to compare chemotherapy and interferon (IFN) in patients with metastatic carcinoid tumors. Patients with documented progressive, unresectable, metastatic carcinoid tumors were randomized between 5 -fluorouracil plus streptozotocin (day 1-5) and recombinant IFN- $\alpha-2 a(3 \mathrm{MU} \times 3$ per week). Primary endpoint was progression-free survival (PFS). From February 1998 to June 2004, 64 patients were included. The two arms were well matched for median age, sex ratio, PS $0-1$, previous chemotherapy, surgery, or radiotherapy. The median PFS for chemotherapy was 5.5 months versus 14.1 for IFN (hazard ratio $=0.75(0.41-1.36)$ ). Overall survival $(O S)$, tolerance, and effects on carcinoid symptoms were not significantly different. Despite a trend in favor of IFN, there was no difference in PFS and OS in advanced metastatic carcinoid tumors and therapeutic effect of both treatments was mild.
\end{abstract}

Endocrine-Related Cancer (2009) 16 1351-1361

\section{Introduction}

Metastatic midgut endocrine carcinomas (formerly called 'carcinoid tumor') are an uncommon neoplasm, accounting for $1 \%$ of digestive cancers. It is often diagnosed late when it has become metastatic (Lepage et al. 2004). Surgery including, when possible, resection of the primary tumor and metastases is the best treatment for metastatic disease (Nave et al. 2001). Transarterial hepatic chemoembolization is used only for nonresectable liver metastases. However, for most patients, only a palliative systemic treatment is appropriate. Somatostatin analogs are useful to control 
hormonal symptoms and have a potential effect on tumor growth. Many chemotherapeutic agents have been evaluated but their effect on survival is unclear. Only four randomized studies have been conducted, and these showed that the best combination (5-fluorouracil (5-FU)-streptozotocin (STZ)) generated response rates from 16 to $33 \%$ (Moertel \& Hanley 1979, Engstrom et al. 1984, Haller et al. 1990, Sun et al. 2005). Biological therapy using human leukocyte interferon (IFN) was investigated in the 1980 s by Oberg et al. (1983). IFN- $\alpha$ primary stabilizes symptoms in functional tumor disease and, in some patients, an antiproliferative effect has been reported. The antitumoral effect is attributed to the inhibition of angiogenesis, induction of apoptosis, and interruption of the cell cycle (Oberg 2000). IFN- $\alpha$ and somatostatin analogs are the main antisecretory drugs used in the treatment of these tumors. In a large study of patients with malignant carcinoid tumors and hepatic metastases receiving IFN- $\alpha$, a biochemical response was obtained in $42 \%$, and stabilization of tumor growth was reported in 39\% (Oberg \& Eriksson 1991). A recent phase II study has suggested that IFN- $\gamma$ was well tolerated, but had no significant antiproliferative effects (Stuart et al. 2004). In contrast, a randomized trial showed that the addition of IFN- $\alpha$ to octreotide may delay tumor growth in patients with midgut carcinoid tumors compared with octreotide alone (Kolby et al. 2003). Only one trial compared the 5 -FU-STZ combination with recombinant IFN- $\alpha$; it showed tumor response rates as high as $50 \%$ in the immunotherapy group. However, this was a limited series that included only 20 patients and no tumor response in the chemotherapy arm was observed (Oberg et al. 1989).

Therefore, because of the potential therapeutic effects of IFN in this disease, a randomized multicenter phase III trial was conducted by the Fédération Nationale des Centres de Lutte Contre le Cancer (FNCLCC) and Fédération Francophone de Cancerologie Digestive (FFCD) among patients with a progressive metastatic endocrine tumor to compare IFN- $\alpha$ to a combination of STZ and 5-FU.

\section{Patients and methods}

\section{Patient selection}

Eligibility criteria were: histologically or cytologically confirmed unresectable malignant carcinoid tumor, i.e. well-differentiated endocrine carcinoma on hematoxylin-eosin staining, with positive Grimelius staining and with chromogranine A expression by immunochemistry; documented tumor progression according to WHO criteria as judged by computed tomography (CT), and/or biochemical progression according to levels of urinary 5-hydroxyindoleacetic acid (5-HIAA; increase in levels of 5-HIAA of more than $25 \%$ ) documented during the 12 months before the enrollment; prior chemotherapy $>6$ weeks; WHO PS of $0-2$; age $>18$ years and $\leq 75$ years; white blood cells (WBC) $>4000 / \mathrm{mm}^{3}$, neutrophils $\geq 2000 / \mathrm{mm}^{3}$, and hemoglobin $\geq 10 \mathrm{~g} / \mathrm{dl}$; platelets $\geq 100000 / \mathrm{mm}^{3}$, serum bilirubin $\leq$ twice the upper limit of normal (ULN), aspartate aminotransferase (AST) $\leq 4$ times ULN, alkaline phosphatase $\leq 4$ times ULN, and serum creatinine $<130 \mu \mathrm{mol} / \mathrm{l}$; proteinuria $<0.5 \mathrm{~g} / 24 \mathrm{~h}$, glycemia $\leq 1.25$ times ULN, and life expectancy $\geq 12$ weeks.

Exclusion criteria were: prior immunotherapy by IFN or other cytokines; prior chemotherapy $<6$ weeks before enrollment; prior radiotherapy to the primary area of measurable disease; myocardial infarction; history of major psychiatric disease; history of epilepsy, brain metastases, and pregnancy.

Written informed consent was obtained from all patients. The protocol was approved by the Regional Ethics Committee (Marseille).

\section{Design of the study and randomization}

\section{Clinical and biochemical work up}

Pre-treatment evaluation included a full medical history, physical examination, hematologic, and biochemical analysis. Radiological (CT) evaluation of the tumor and $24 \mathrm{~h}$ collection of urine for 5-HIAA were also performed.

After confirming the eligibility criteria, all registered patients were randomized 1:1 through a minimization program at the FFCD center (Dijon, France) by fax or phone: in arm A, 5-FU plus STZ, or in arm B recombinant IFN- $\alpha$-2a (IFN; ROFERON supplied by Roche).

Patients were stratified by WHO PS $(0,1$, or 2$)$, prior chemotherapy and/or immunotherapy (yes or no), and participating institutions.

\section{Treatment plan}

In arm A, chemotherapy was administered i.v. using a central catheter. Every 6-week cycle consisted of five administrations of 5 -FU ( $400 \mathrm{mg} / \mathrm{m}^{2}$ per day) by $2 \mathrm{~h}$ i.v. infusion (day 1-5), in combination with five administrations of STZ $\left(500 \mathrm{mg} / \mathrm{m}^{2}\right.$ per day) by $2 \mathrm{~h}$ i.v. infusion (day 1-5). In arm B, IFN- $\alpha$ (ROFERON) was administered continuously at doses of $3 \times 10^{6}$ three times a week by s.c. injection (6-week cycle). 
In each arm, treatment was planned for at least 12 weeks (two cycles) and for a maximum of eight cycles. After eight cycles, patients experiencing stabilization of the disease or an objective response pursued the allocated therapy. Treatment was stopped in case of disease progression (tumoral or biochemical), limiting toxicities, or patient request. Then further treatments were chosen by the referent clinician including the possibility of cross over. Surgical or radiotherapy treatments were allowed after an objective response and evaluation of the toxicity.

Concomitant treatments with somatostatin analogs were allowed in patients suffering from significant carcinoid symptoms. In arm $\mathrm{A}$, corticoids and setron were advised to prevent nausea. In arm B, paracetamol was advised to treat flu-like syndrome if necessary.

\section{Dose adjustment}

At the first occurrence of grade 3 or 4 toxicity, treatment was interrupted until recovery to grade 2 toxicity or less. Then treatment was pursued with a $50 \%$ reduction in the original dose. A second occurrence of any grade 3 or 4 toxicity led to a further $50 \%$ reduction. Any dose reduction was permanent. No dose escalation was permitted. Treatment was stopped if the patient had not recovered to grade 2 toxicity or less within 6 weeks or if other grade 3-4 toxicities occurred. STZ was stopped in case of proteinuria $>0.5 \mathrm{~g} / 24 \mathrm{~h}$ or creatinemia $>200 \mu \mathrm{mol} / \mathrm{l}$.

\section{Evaluation and follow up}

After randomization, the patients had a complete clinical examination every 6 weeks during which the following were measured: WHO PS; weight; number of stools/day; number of flush/day during the week before; laboratory investigations (platelets, WBC, hemoglobin, creatinine, glycemia, biochemical markers, transaminases, alkaline phosphatases, bilirubin and proteinuria).

Radiological assessment and biochemical evaluation including 5-HIAA levels and enolase neuro specific (NSE) were performed every 12 weeks. Based on CT imaging, the tumor response was classified according to WHO criteria: complete response was defined as a complete disappearance of all tumor lesions; partial response was defined as a 50\% reduction in the product of perpendicular tumor diameters without the appearance of new metastases; stable disease was defined as $<50 \%$ reduction, but no $>25 \%$ increase in the product of perpendicular diameters; and progressive disease was defined as more than a $25 \%$ increase in the product of perpendicular diameters or the appearance of new metastases. The biochemical response was defined as more than a $50 \%$ reduction in the initial 5-HIAA level (mean value in two urine samples collected in a $24 \mathrm{~h}$ period) lasting for 1 month. Biochemical progression was defined as more than a $25 \%$ increase in 5-HIAA level.

All toxicities were graded according to WHO criteria every 6 weeks. Serious adverse events were also recorded within $24 \mathrm{~h}$.

After ending the treatment, follow up information was available for all patients every 12 weeks until progression or death.

\section{Statistical methods}

This phase III study was designed to evaluate 1-year progression-free survival (PFS) as the primary endpoint. It was planned to include 120 patients, to observe 38 progressions or deaths, in order to detect an expected improvement in 1-year PFS from 55\% (Engstrom et al. 1984) to $80 \%$ (bilateral $\alpha=5 \%$ and $\beta=20 \%$ ) in the IFN arm. The trial was closed early, after enrollment of 64 patients, due to insufficient recruitment. However, with 46 observed deaths or progression at the data cut off, the plan number of events was achieved. All analyses were performed on a strict intent to treat principle (all included patients whatever the eligibility criteria and treatment received). As exploratory analyses, we have also performed a per-protocol analysis of PFS, time to progression (TTP), and WHO tumor response among midgut primary localization patients and excluding patients who have received 5-FU-STZ before randomization and patients who did not receive at least one cycle of treatment.

Qualitative and continuous variables have been described using percent and mean (s.D.) and median (minimum-maximum) respectively, and then compared using the $\chi^{2}$ or Fisher exact test and the Mann and Whitney nonparametric test respectively.

The PFS was defined as the time between randomization and the first sign of progression (biochemical or tumoral according WHO criteria) or death. Patients alive without progression were censored at the last follow up. For exploratory purpose, we have also studied PFS definition including only tumoral progression (WHO criteria) or death.

Secondary endpoints were: the TTP defined as the time between randomization and the first sign of biochemical or tumoral progression; patients who were alive or had died without progression were censored at the last follow up. Overall survival (OS) was defined as the time between randomization and death (all causes). 
Tolerance and carcinoid symptoms were also studied. Median follow up was calculated according to reverse Kaplan-Meier estimates.

Survival curves were plotted using Kaplan-Meier estimates and were described using median $(95 \% \mathrm{CI})$ and 1- and 2-year survival rates. Survival curves were compared using log-rank tests. A univariate Cox model was used to calculate the hazard ratio (HR) with a $95 \%$ CI. No interim analysis was planned. All analyses were performed using Stata software (v8, College Station, TX, USA) at the 0.05 level of significance.

\section{Results}

Between February 1998 and June 2004, 64 patients (35 men and 29 women) were included in 23 centers: 32 patients in each arm. One patient in the chemotherapy arm had already received one cycle of IFN. While major eligibility criteria were respected, 13 patients and 14 patients did not meet minor biochemical or hematological eligibility criteria in arm A and arm B respectively, and three patients were lost of follow up in $\operatorname{arm~B.~However,~the~} 64$ patients were included in the intent to treat analyses (Fig. 1). At the cut off date, the median follow up was 46 months (range 1-83).

\section{Patients' characteristics}

Patients' characteristics are summarized in Table 1. The mean age was 60 years (s.D. 10 years). The WHO performance status $0-1$ was 88 and $85 \%$ in the chemotherapy and IFN arms respectively. Among the 64 patients randomized, $40(63 \%)$ were midgut carcinoid (72\% in the chemotherapy arm and $53 \%$ in the IFN arm), 2 (6\%) were hindgut carcinoid (one in each arm), and 9 were foregut carcinoid (12\% in the chemotherapy arm and $15 \%$ in the IFN arm); other locations had elevated 5-HIAA with or without carcinoid symptoms at the study entry. Out of 64 patients, 63 had progressive and measurable disease according to WHO criteria during the 12 months before inclusion; only one patient had biochemical progression only and no measurable target. Arms A and B were well matched for age, sex, biochemical

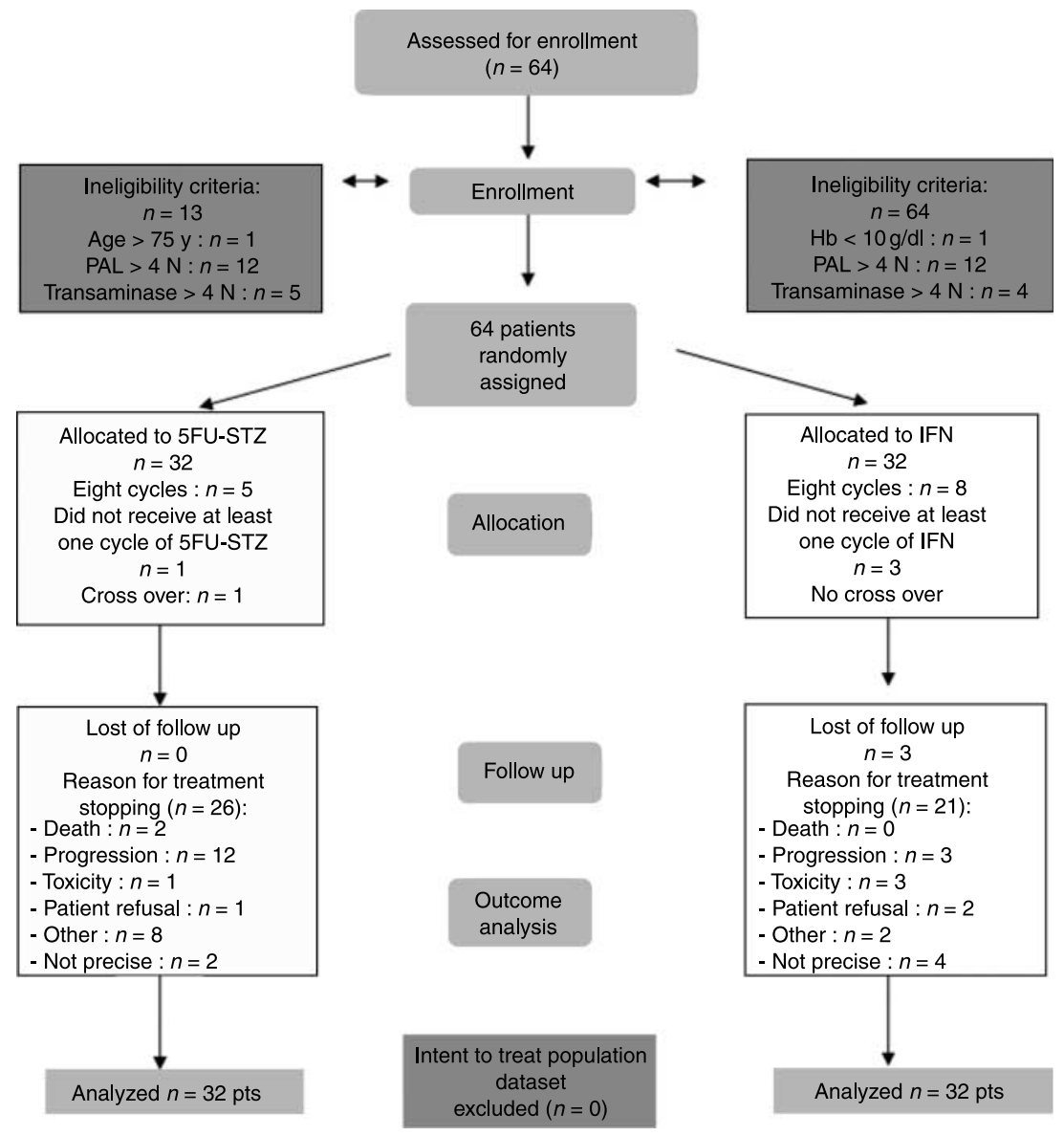

Figure 1 Consort diagram of the study 
Table 1 Patients' demographics and clinical characteristics

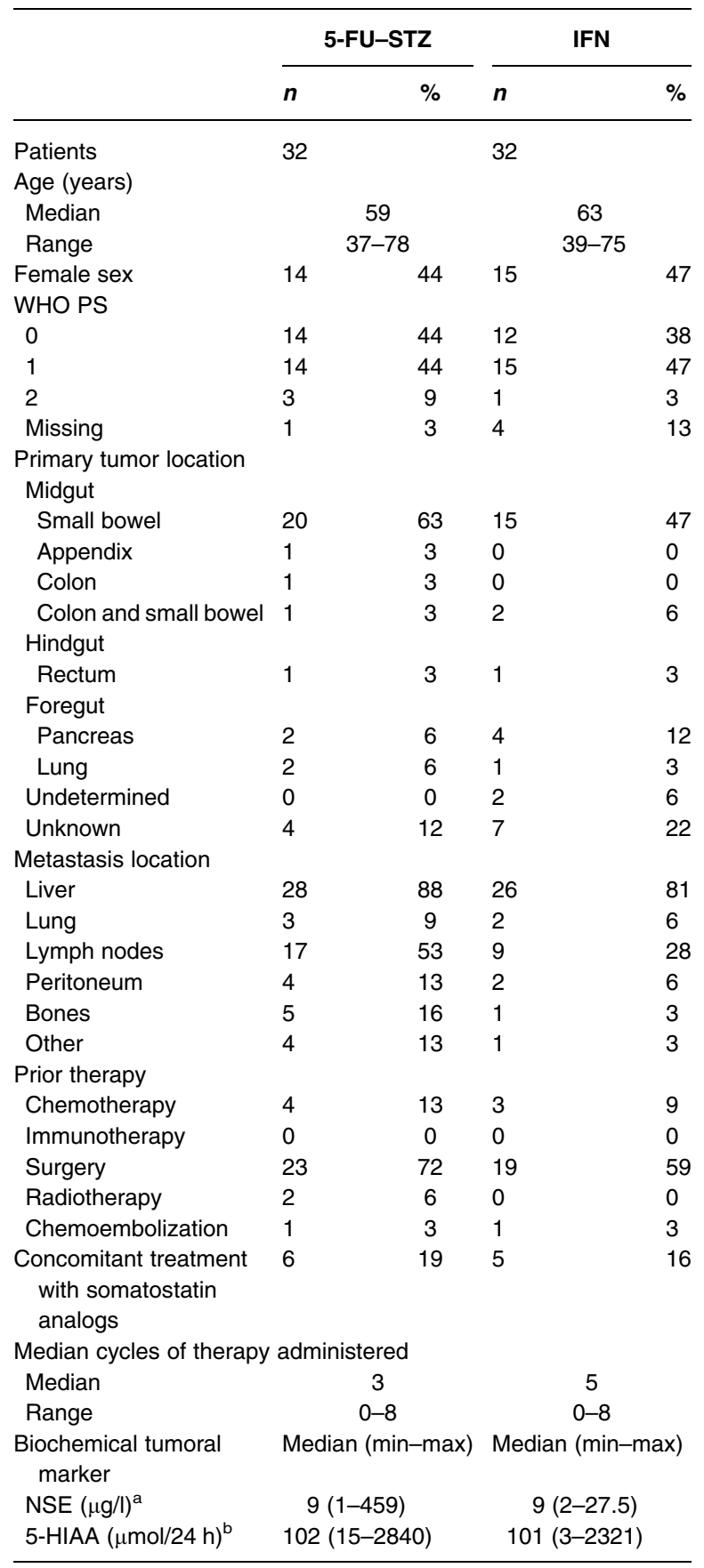

${ }^{a_{\text {normal }}}$ value $<12.5 \mu \mathrm{g} / \mathrm{L}$.

${ }^{b}$ normal value $<47 \mu \mathrm{mol} / 24 \mathrm{~h}$.

markers, prior treatments (chemotherapy, immunotherapy, and surgery), and the presence of liver metastases. However, lymph node metastases were more frequent in the 5-FU-STZ arm $(P=0.012)$. Four patients (13\%) in arm A and three patients (9\%) in arm $\mathrm{B}$ had received chemotherapy more than 6 weeks before enrollment. Among them, five patients received
5-FU-STZ (three in arm A and two in arm B) but they had stable disease, and chemotherapy was stopped more than 6 weeks before enrollment. Others had received 5-FU monotherapy. At inclusion, seven patients in arm $\mathrm{A}$ and nine patients in arm $\mathrm{B}$ had normal level of urinary 5-HIAA.

In each arm, one patient (3\%) had undergone hepatic transarterial chemoembolization for liver metastases before randomization.

\section{Treatment delivery}

The median number of IFN cycles was 5 in arm B; three patients did not receive at least one cycle of IFN and eight patients received eight cycles. In arm A, the median number of cycle of 5-FU-STZ was 3; one patient did not receive at least one cycle of 5-FU-STZ and five patients received eight cycles.

\section{Toxicity}

The distribution of maximum grade toxicities for each arm is shown in Table 2. There were no significant differences in grade 3-4 toxicities between two arms. In contrast, the occurrence of (grade 1-4) hematological toxicities and fever was more frequent in the IFN arm $(P<0.05)$, whereas renal toxicity (grade $1-2$ proteinuria) and nausea were more frequent in the FU-STZ arm $(P<0.05)$.

\section{Carcinoid symptoms}

Both chemotherapy and IFN- $\alpha$ gave a similar degree of control of carcinoid symptoms during treatments (Table 2). During treatment cycles, the mean number of stools per day during the week preceding the cycle was 2.1 (s.D. 1.4) and 2.3 (s.D. 2.0) in arm A and B respectively, while the mean number of episodes of flushing per day in the week preceding the cycle was 1 (s.D. 2.0) and 0.5 (s.D. 1.2) in arm A and B respectively, but treatment with somatostatin analogs was allowed (octreotide $30 \mathrm{mg} / \mathrm{month}$ ). After two cycles of treatment, the mean of stools per day had decrease in the 5-FU-STZ arm, while it was similar in the IFN arm respectively $(P=0.0721)$ : -0.78 (s.D. 1.37) and 0.06 (s.D. 1.21). After two cycles, the mean number of episodes of flushing per day had decrease in the 5-FU-STZ arm and in the IFN arm respectively $(P=0.26):-0.50$ (s.D. 1.83$)$ and -0.50 (s.D. 1.03$)$.

\section{Best response according to WHO criteria}

There was no complete response in either arm. In the chemotherapy arm, one patient $(3 \%)$ had a partial response, while three patients $(9 \%)$ had a partial 
Table 2 Treatment-related toxicities according to WHO criteria and carcinoid symtoms according to treatment arm

\begin{tabular}{|c|c|c|c|c|c|c|}
\hline \multirow[b]{2}{*}{ Toxicities } & \multicolumn{3}{|c|}{ Grade 1-4 } & \multicolumn{3}{|c|}{ Grade 3-4 } \\
\hline & 5-FU-STZ $(n=32)$ & IFN $(n=32)$ & $P^{a}$ & $5-F U-S T Z(n=32)$ & IFN $(n=32)$ & $P^{a}$ \\
\hline Anemia & $2(6 \%)$ & $9(28 \%)$ & 0.037 & $2(6 \%)$ & $1(3 \%)$ & NS \\
\hline Leukopenia & $3(9 \%)$ & $14(44 \%)$ & 0.001 & $0(0 \%)$ & $0(0 \%)$ & NS \\
\hline Neutropenia & $4(13 \%)$ & $14(44 \%)$ & 0.003 & $0(0 \%)$ & $1(3 \%)$ & NS \\
\hline Thrombocytopenia & $3(9 \%)$ & $4(13 \%)$ & NS & $1(3 \%)$ & $0(0 \%)$ & NS \\
\hline Overall hematological toxicities & $10(31 \%)$ & $20(63 \%)$ & 0.003 & $3(9 \%)$ & $2(6 \%)$ & NS \\
\hline Creatininemia & $6(19 \%)$ & $2(6 \%)$ & NS & $0(0 \%)$ & $0(0 \%)$ & NS \\
\hline Proteinuria & $13(41 \%)$ & $2(6 \%)$ & 0.001 & $0(0 \%)$ & $0(0 \%)$ & NS \\
\hline Nausea & $16(50 \%)$ & $7(22 \%)$ & 0.044 & $2(6 \%)$ & $1(3 \%)$ & NS \\
\hline Vomiting & $9(28 \%)$ & $5(16 \%)$ & NS & $2(6 \%)$ & $1(3 \%)$ & NS \\
\hline Diarrhea & $14(44 \%)$ & $17(53 \%)$ & NS & $2(6 \%)$ & $2(6 \%)$ & NS \\
\hline Fever & $2(6 \%)$ & $8(25 \%)$ & 0.028 & $0(0 \%)$ & $1(3 \%)$ & NS \\
\hline Asthenia & $21(66 \%)$ & $19(59 \%)$ & NS & $3(9 \%)$ & $3(9 \%)$ & NS \\
\hline Pain & $18(56 \%)$ & $17(53 \%)$ & NS & $2(6 \%)$ & $1(3 \%)$ & NS \\
\hline Overall toxicities & $29(91 \%)$ & 27 (84\%) & NS & $11(34 \%)$ & $8(25 \%)$ & NS \\
\hline \multicolumn{2}{|l|}{$\begin{array}{l}\text { Carcinoid symptoms during } \\
\text { treatments }\end{array}$} & Mean (S.D.) & & \multicolumn{2}{|l|}{ Mean (s.D.) } & $P^{\mathrm{b}}$ \\
\hline \multicolumn{2}{|c|}{$\begin{array}{l}\text { Number of episodes of flushing per day } \\
\text { during week before }\end{array}$} & $1.0(2.1)$ & & \multicolumn{2}{|l|}{$0.5(1.2)$} & NS \\
\hline \multicolumn{2}{|c|}{ Cycle 1} & $1.2(2.3)$ & & \multicolumn{2}{|l|}{$0.8(1.8)$} & NS \\
\hline \multicolumn{2}{|l|}{ Cycle 2} & $0.8(1.4)$ & & \multicolumn{2}{|l|}{$0.5(1.3)$} & NS \\
\hline \multicolumn{2}{|l|}{ Cycle 3} & $0.3(0.8)$ & & \multicolumn{2}{|l|}{$0.5(1.2)$} & NS \\
\hline \multicolumn{2}{|l|}{ Cycle 4} & $0.3(1.1)$ & & \multicolumn{2}{|l|}{$0.6(1.3)$} & NS \\
\hline \multicolumn{2}{|l|}{ Cycle 5} & $0.3(0.7)$ & & \multicolumn{2}{|l|}{$0.7(1.4)$} & NS \\
\hline \multicolumn{2}{|l|}{ Cycle 6} & $0.1(0.3)$ & & \multicolumn{2}{|l|}{$0.2(0.6)$} & NS \\
\hline \multicolumn{2}{|l|}{ Cycle 7} & $0.6(0.9)$ & & \multicolumn{2}{|l|}{$0.2(0.4)$} & NS \\
\hline \multicolumn{2}{|l|}{ Cycle 8} & $0.3(0.5)$ & & \multicolumn{2}{|l|}{$0.3(0.5)$} & NS \\
\hline \multicolumn{2}{|c|}{ Number of stool per day during week before } & $2.1(1.4)$ & & \multicolumn{2}{|l|}{$2.3(2.0)$} & NS \\
\hline \multicolumn{2}{|c|}{ Cycle 1} & $2.2(1.8)$ & & \multicolumn{2}{|l|}{$2.4(2.7)$} & NS \\
\hline \multicolumn{2}{|l|}{ Cycle 2} & $2.5(2.3)$ & & $2.3(1.9)$ & & NS \\
\hline Cycle 3 & & $1.7(1.1)$ & & $2.3(2.2)$ & & NS \\
\hline Cycle 4 & & $1.3(0.9)$ & & $2.2(2.1)$ & & NS \\
\hline Cycle 5 & & $2.1(2.2)$ & & $2.2(2.2)$ & & NS \\
\hline Cycle 6 & & $2.2(2.2)$ & & 1.7 (1.3) & & NS \\
\hline Cycle 7 & & $1.5(1.7)$ & & $1.8(1.8)$ & & NS \\
\hline Cycle 8 & & $2.3(2.1)$ & & $1.6(1.0)$ & & NS \\
\hline
\end{tabular}

${ }^{\mathrm{a}}$ Fisher exact test.

${ }^{\mathrm{b}}$ Mann and Whitney test.

response in the IFN arm. The rates of stable disease and response were $56 \%$ (18 patients) and 63\% (20 patients) in the chemotherapy and IFN arm respectively $(P=0.12$; Table 3$)$. Disease control was achieved in 19 patients (59\%) in the chemotherapy arm and 23 patients (72\%) in the IFN arm. Among the four patients in partial response according to WHO criteria, three had small bowel primary localization (one in 5-FU-STZ and two in IFN arm) and one patient had an undetermined primary localization.

In per-protocol analysis (among 36 midgut patients receiving at least one cycle of treatment and who did not have received 5-FU-STZ before randomization), in the chemotherapy arm, one patient (5\%) had a partial response, while two patients (13\%) had a partial response in the IFN arm. The rates of stable disease and response were $60 \%$ (12 patients) and 75\% (12 patients) in the chemotherapy and IFN arm respectively $(P=0.31)$. Disease control was achieved in 13 patients $(65 \%)$ in the chemotherapy arm and 14 patients (88\%) in the IFN arm.

\section{Progression-free survival}

In the chemotherapy arm, 14 (43\%) patients had progression according to WHO criteria and 7 patients had biochemical progression only (22\%), 6 patients died without progression, and 5 patients were alive without 
Table 3 Response to therapy according to treatment group

\begin{tabular}{|c|c|c|c|c|c|c|}
\hline & \multicolumn{2}{|c|}{ 5-FU-STZ $(N=32)$} & \multicolumn{3}{|c|}{ IFN $(N=32)$} & \multirow[b]{2}{*}{$\boldsymbol{P}$} \\
\hline & $N$ & $\%$ & $N$ & & $\%$ & \\
\hline \multicolumn{7}{|l|}{ Best response therapy according to $\mathrm{WHO}$ criteria } \\
\hline Complete & 0 & 0 & 0 & & 0 & \\
\hline Partial & 1 & 3 & 3 & & 9 & \\
\hline Stability & 18 & 56 & 20 & & 63 & \\
\hline Progression & 10 & 31 & 3 & & 9 & \\
\hline Missing & 3 & 9 & 6 & & 19 & \\
\hline \multicolumn{7}{|l|}{ Tumor progression at data cut off } \\
\hline WHO criteria & 14 & 43 & 10 & & 31 & \\
\hline Biochemical progression only & 7 & 22 & 3 & & 9 & \\
\hline No progression & 9 & 28 & 14 & & 44 & \\
\hline Missing & 2 & 6 & 5 & & 16 & \\
\hline Death & 16 & 50 & 13 & & 41 & \\
\hline Progression-free survival (PFS) & \multicolumn{2}{|c|}{$n=32$} & \multicolumn{3}{|c|}{$n=32$} & \\
\hline Median PFS in months $(95 \% \mathrm{Cl})$ & \multicolumn{2}{|c|}{$5.5(2.9-25)$} & \multicolumn{3}{|c|}{$14.1(6.7-21.2)$} & 0.34 \\
\hline 1-year PFS rate $(95 \% \mathrm{Cl})$ & \multicolumn{2}{|c|}{$44 \%(26-60)$} & \multicolumn{3}{|c|}{$51 \%(31-68)$} & \\
\hline 2-year PFS rate $(95 \% \mathrm{Cl})$ & \multicolumn{2}{|c|}{$34 \%(18-50)$} & \multicolumn{3}{|c|}{$26 \%(11-44)$} & \\
\hline Progression-free survival (PFS) according WHO criteria & \multicolumn{2}{|c|}{$n=32$} & \multicolumn{3}{|c|}{$n=32$} & \\
\hline Median PFS $(95 \% \mathrm{Cl})$ & \multicolumn{2}{|c|}{$7.3(3.0-25.0)$} & \multicolumn{3}{|c|}{$14.1(7.6-\mathrm{NR})$} & 0.25 \\
\hline 1-year PFS (95\% CI) & \multicolumn{2}{|c|}{$46 \%(28-62)$} & \multicolumn{3}{|c|}{$53 \%(32-70)$} & \\
\hline 2-year PFS (95\% Cl) & \multicolumn{2}{|c|}{$35 \%(19-52)$} & \multicolumn{3}{|c|}{$33 \%(14-52)$} & \\
\hline Time to progression (TTP) & \multicolumn{2}{|c|}{$n=32$} & \multicolumn{3}{|c|}{$n=32$} & \\
\hline Median TTP in months $(95 \% \mathrm{Cl})$ & \multicolumn{2}{|c|}{$8.5(2.9-60.4)$} & \multicolumn{3}{|c|}{$19.6(6.7-N R)$} & 0.21 \\
\hline 1-year TTP cumulative rate $(95 \% \mathrm{Cl})$ & \multicolumn{2}{|c|}{$52 \%(35-70)$} & \multicolumn{3}{|c|}{$38 \%(23-60)$} & \\
\hline 2-year TTP cumulative rate $(95 \% \mathrm{Cl})$ & \multicolumn{2}{|c|}{$55 \%(39-74)$} & \multicolumn{3}{|c|}{$55 \%(36-77)$} & \\
\hline Overall survival (OS) & \multicolumn{2}{|c|}{$n=32$} & \multicolumn{3}{|c|}{$n=32$} & \\
\hline Median OS in months $(95 \% \mathrm{Cl})$ & & & & $44.3(23-59)$ & & 0.83 \\
\hline 1-year OS & & & & $89 \%(68-96)$ & & \\
\hline 2-year OS & & & & $70 \%(47-85)$ & & \\
\hline
\end{tabular}

progression (Table 3). In the IFN arm, ten (31\%) patients had progression according to WHO criteria and three patients had biochemical progression only (9\%), six patients died without progression, and ten patients were alive without progression (Table 3).

Statistically significant difference in PFS was not observed according to the treatment arm (log-rank $P=0.34$; Fig. 2a). However, the median PFS was 5.5 months (95\% CI: 2.9-25.0) in the chemotherapy arm and 14.1 months (95\% CI: 6.7-21.2) in the IFN arm, and 1-year PFS was respectively $44 \%(95 \% \mathrm{CI}$ : 26-60\%) and 51\% (95\% CI: 31-68\%). The univariate Cox HR was 0.75 (95\% CI: 0.41-1.36). However, there is a clinically relevant difference in PFS, but this trial has not sufficient power to detect it.

In per-protocol analysis (among 36 midgut patients receiving at least one cycle of treatment and who did not have received 5-FU-STZ before randomization), the median PFS was 8.5 months (95\% CI: $2.8-43.6$ ) in the chemotherapy arm and 14.1 months $(95 \% \mathrm{CI}$ : 7.6-NR) in the IFN arm (log-rank $P=0.42)$, and 1-year PFS was respectively 50\% (95\% CI: 27-69\%) and 56\% (95\% CI: $30-76 \%$ ).

\section{Progression-free survival according to WHO criteria only}

Statistically significant difference in PFS was not observed according to the treatment arm (log-rank $P=0.25$; Fig. 2b). However, the median PFS was 7.3 months (95\% CI: 3.0-25.0) in the chemotherapy arm and 14.1 months (95\% CI: 7.6-NR) in the IFN arm, and 1-year PFS was respectively $46 \%$ (95\% CI: $28-62 \%$ ) and 53\% (95\% CI: 32-70\%). The univariate Cox HR was 0.67 (95\% CI: 0.35-1.32).

In per-protocol analysis, the median PFS was 13 months (95\% CI: 2.8-43.6) in the chemotherapy arm and 19.7 months (95\% CI: 9.5-NR) in the IFN arm (log-rank $P=0.31$ ), and 1-year PFS was respectively $54 \%$ (95\% CI: $30-73 \%)$ and $61 \%(95 \%$ CI: $32-80 \%)$.

\section{Time to progression}

There was no significant difference in TTP according to the treatment arm (log-rank $P=0.21$; Fig. 3). However, the median TTP (patients free of progression) was 8.5 months (95\% CI: 2.9-60.4) in the 


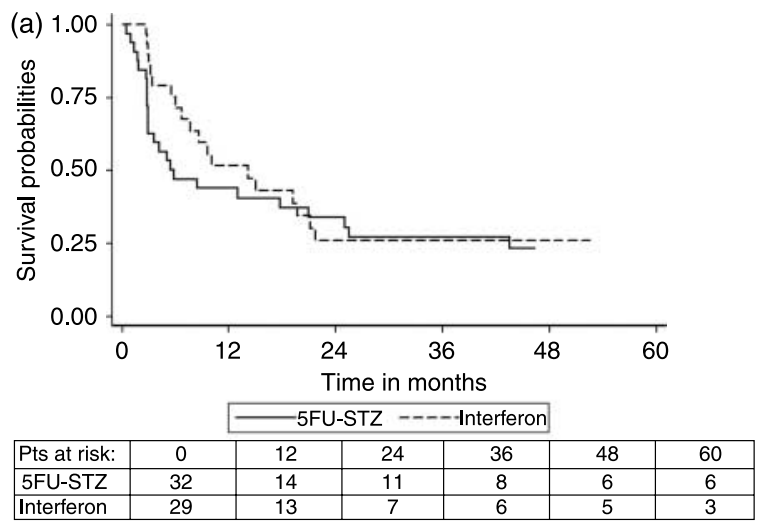

Log-rank $P=0.34$

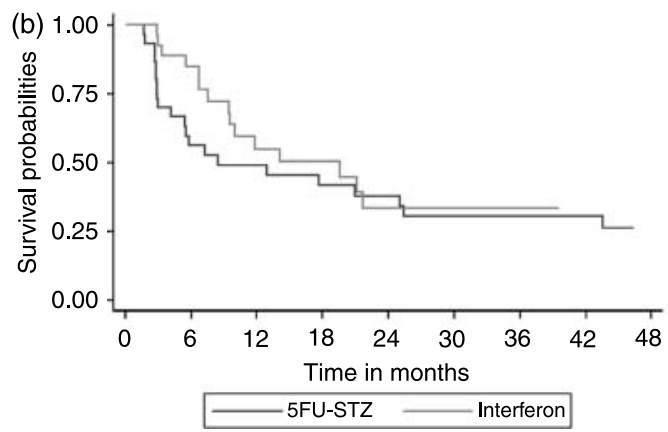

Number at risk

\begin{tabular}{|l|l|l|l|l|l|l|l|l|l|}
\hline 5FU-STZ & 30 & 16 & 13 & 11 & 10 & 7 & 7 & 7 & 0 \\
\hline Interferon & 27 & 21 & 12 & 10 & 6 & 5 & 5 & 0 & 0 \\
\hline
\end{tabular}

Log-rank $P=0.32$

Figure 2 (a) Progression-free survival (WHO criteria or biochemical progression or death) according to treatment arm (Kaplan-Meier estimation). (b) Progression-free survival (WHO criteria or death) according to treatment arm (Kaplan-Meier estimation).

chemotherapy arm and 19.6 months (95\% CI: 6.7-NR) in the IFN arm (Table 3), and 1-year TTP was 52\% (95\% CI: $35-70 \%$ ) and $38 \%$ (95\% CI: $23-60 \%)$ respectively. The univariate Cox HR was $0.63(95 \%$ CI: 0.31-1.30).

In per-protocol analysis (amongst 36 midgut patients receiving at least one cycle of treatment and who did not have received 5-FU-STZ before randomization), the median TTP was 13.2 months (95\% CI: 2.9-60.4) in the chemotherapy arm and 19.2 months $(95 \% \mathrm{CI}$ : 7.6-NR) in the IFN arm (log-rank $P=0.29)$, and 1-year TTP was respectively 53\% (95\% CI: $29-72 \%)$ and $69 \%$ (95\% CI: $40-86 \%)$.

\section{Overall survival}

Out of 29 patients, $16(50 \%)$ patients in the chemotherapy arm and $13(41 \%)$ patients in the immunotherapy arm died respectively. As shown in

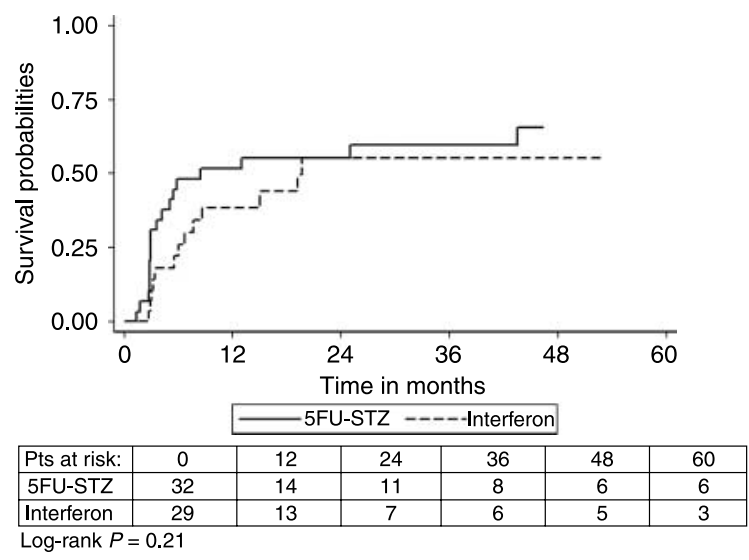

Figure 3 Time to progression (WHO criteria or biochemical progression) according to treatment arm.

Table 3, OS did not differ according to treatment (log-rank $P=0.83)$. Median OS was $30.4(21-\mathrm{NR})$ and 44 months (23-59) respectively (Fig. 4).

The 1-year OS rate was $81 \%(63-91 \%)$ in arm A and $89 \%(68-96 \%)$ in arm B, and the 2-year OS rate was $70 \%$ in the two groups. The univariate Cox HR was $0.92(0.44-1.92)$.

\section{Discussion}

Patients with midgut carcinoid tumor often present with widespread disease at the time of diagnosis, and curative surgery is therefore seldom possible. The introduction of somatostatin analogs has improved the management of carcinoid syndrome. However, because of generally low rates of response, there is still no standard systemic chemotherapy for patients with metastatic carcinoid tumors. On the basis of randomized published studies (Moertel \& Hanley 1979, Engstrom et al. 1984, Haller et al. 1990,

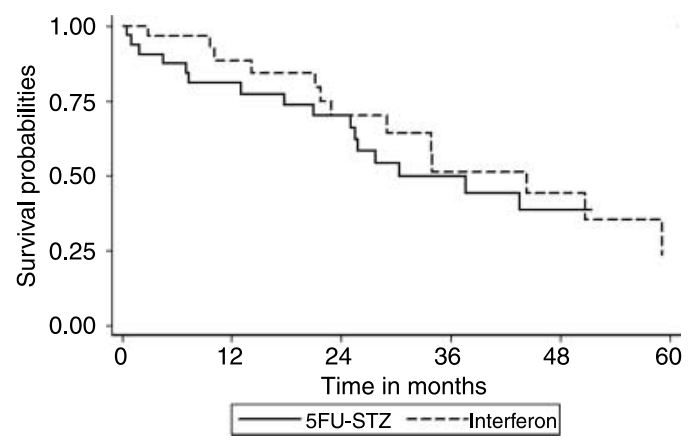

\begin{tabular}{|l|c|c|c|c|c|c|}
\hline Pts at risk: & 0 & 12 & 24 & 36 & 48 & 60 \\
\hline 5FU-STZ & 32 & 23 & 19 & 11 & 7 & 6 \\
\hline Interferon & 29 & 22 & 14 & 9 & 7 & 3 \\
\hline
\end{tabular}
Log-rank $P=0.83$

Figure 4 Overall survival according to treatment arm. 
Sun et al. 2005), the efficacy of a combination of STZ and fluoropyrimidine is modest, but, in the absence of other active chemotherapy regimen, this combination has been widely used for the treatment of advanced carcinoid tumors in case of progressive disease (Conseil Scientifique de la FFCD 2003). It has been shown that this regimen has an acceptable toxicity profile in the management of metastatic carcinoids (Gonzalez et al. 2003). Moreover, 5-FU-STZ combination was still a recommended option at the end of the study for patients with extrahepatic metastasis or hepatic metastasis after failure of transarterial hepatic chemoembolization even if the recent ENETS Consensus Conference did not recommend chemotherapy in midgut tumors (Eriksson et al. 2008).

IFN has proven antiproliferative effects in some tumors, including endocrine tumors. Recently, Detjen et al. (2000) showed the direct effects of IFN- $\alpha$ on the cell cycle with a prolongation of the $S$ phase in neuroendocrine cells. The increase in fibrotic tissue or an antiangiogenic effect might be other mechanisms responsible for the antiproliferative effects of IFN- $\alpha$ (Dirix et al. 1991, Oberg 1992). However, no previous phase III trial, comparing chemotherapy with IFN- $\alpha$ on survival, has been reported. IFN dose used in this trial was chosen according to previous studies. Indeed, studies with increased dose have shown bad tolerance (Pavel et al. 2006).

Owing to the low incidence of carcinoid tumors and their heterogeneity, it is always difficult to conduct prospective trial in this disease, and this was the main reason for our study to fail to reach the planned inclusion. However, due to the long duration of the trial resulting in a median follow up of near 4 years, the number of required events for the planned power was achieved with 46 observed deaths or progressions. Our trial failed to demonstrate a significant PFS advantage because our initial hypothesis regarding differences in PFS was not reached with an observed HR of 0.75 instead of an expected HR of 0.37 resulting in about $20 \%$ power. This trial is the first phase III multicenter trial, which has compared recombinant IFN- $\alpha$ with a combination of 5-FU-STZ in a relatively large number of patients selected on strict criteria. Before entering this trial, all patients had to have a histologically proven metastatic carcinoid with a documented tumor progression in the preceding year. There was some evidence of activity in the two treatments arms, but no difference between them in term of response rate, stabilization of the disease, PFS, and OS.

There was a trend in favor of a better TTP and PFS for the IFN- $\alpha$ arm, which translated in a nonsignificant survival advantage in favor of IFN- $\alpha$. Thus, we may conclude from our study that IFN may be considered as an active treatment and an alternative to chemotherapy for patients with carcinoid tumors and disease progression. Specifically, we observed that more patients had no progression in the IFN arm, and this effect may be due to the inhibitory effect of IFN on the growth of midgut carcinoid tumors.

In contrast to previous published trials, the response rate was very low (3\%) in the chemotherapy arm but no severe toxicities, specially renal toxicity, were observed (Moertel \& Hanley 1979, Engstrom et al. 1984, Haller et al. 1990, Sun et al. 2005). Among three recent randomized studies comparing a combination of IFN plus somatostatin analogs (octreotide or lanreotide) versus somatostatin analogs alone, two failed to show a better antiproliferative effect of the combination (Faiss et al. 2003, Arnold et al. 2005). In contrast, the third, including only midgut carcinoid tumors, has shown a significantly reduced risk of tumor progression for patients treated with IFN- $\alpha$ (Kolby et al. 2003).

Our study failed to show a superiority of IFN; this result can probably be explained by the absence of arm without treatment and the sample size. Moreover, we have more midgut tumors in the chemotherapy arm, and we know the less favorable prognosis of foregut and hindgut tumors compared with midgut tumors. Both IFN and chemotherapy control disease progression in about two-third of patients.

Moreover, IFN and chemotherapy demonstrated a similar degree of control for the carcinoid symptom, but treatment with somatostatin analogs was allowed, and the frequency of diarrhea and flushing was low; this could underestimate the effect of IFN on symptoms. Regarding overall toxicity and tolerance to the treatments, there was no significant difference between the two arms for grade 3 and 4 side effects. However, the toxicity profile was different, and the median number of cycles of therapy was higher in the immunotherapy arm. Diarrhea might be a symptom of the carcinoid syndrome and cannot be attributed to the treatment only. Moreover, concurrent treatment with octreotide, for which a cytostatic effect has been reported in the PROMID study, could be a potential confounder in trials, but, in our study, the rate of concomitant treatment with somatostatin analogs was similar in both arms (Arnold et al. 2009).

In summary, based on our study, immunotherapy with IFN- $\alpha$ as well as a chemotherapy using a combination of 5-FU-STZ induces very few objective responses ( 9 and $3 \%$ respectively), but is able to stop disease progression in about two-third of patients (72 and 59\% respectively). Moreover, there was no significant difference between the two treatments for 
side effects and for control of carcinoid symptoms. However, the low frequency of symptoms in the cohort is also reflected by the use of somatostatin analogs, and a comparison of both strategies with respect to symptom control is certainly biased. Despite any significant improvement in PFS and OS as compared to chemotherapy (FU-STZ), there was a trend towards a better PFS for the IFN arm, even if this potential benefit was not significant and nor did it translate into a significant survival advantage. Considering the therapeutic effects, adverse reactions, and simplicity of administration, IFN- $\alpha$ is a reasonable alternative in the treatment of patients with malignant carcinoid tumors.

A recent study on 17 patients with neuroendocrine gastropancreatic carcinomas showed that peg IFN- $\alpha$ provides symptomatic and antiproliferative efficacy and is better tolerated than IFN- $\alpha$ (Pavel et al. 2006). It is probably easier to administer a new pegylated form, and comparative prospective trials of peg IFN- $\alpha$ and non-pegylated IFN- $\alpha$ are warranted in this indication. Recent promising results with new targeted therapies like vascular endothelial growth factor (VEGF) and mammalian target of rapamycin (mTOR) inhibitors could offer new therapeutics options (Moreno et al. 2008, Yao et al. 2008).

In conclusion, our trial suggests that both IFN and chemotherapy combining 5-FU/STZ may be considered as therapeutic options in patients with progressive and disseminated midgut carcinoid tumors. Despite a trend in favor of IFN, no significant difference in PFS and OS was observed between the two treatment groups. To demonstrate a beneficial effect on PFS with an expected HR of 0.75 , a larger phase III study should be required with new targeted therapies.

\section{Declaration of interest}

We declare that there is no conflict of interest that could be perceived as prejudicing the impartiality of the research reported.

\section{Funding}

This research did not receive any specific grant from any funding agency in the public, commercial, or not-for-profit sector. Final responsibility for the decision to submit for publication: L Dahan and F Bonnetain.

\section{Author contribution statement}

Manuscript writing: L Dahan and F Bonnetain contributed equally to this work. Review: E Mitry, J L Raoul, B Coudert, and Ph Rougier. Data management: F Ricard. Protocol writing: J F Seitz, P Rougier, J L Raoul, L Dahan, and F Bonnetain. Investigators: L Dahan, P Rougier, J-L Raoul, E Gamelin, P-L Etienne, G Cadiot, E Mitry, D Smith, F Cvitkovic, B Coudert, L Bedenne, and J-F Seitz. Administrative support: L Bedenne.

\section{Acknowledgements}

Presented at the 42nd annual meeting of the American Society of Clinical Oncology, June 2-6, 2006, Atlanta and at the 9th World Congress on Gastrointestinal Cancer, June 27-30, 2007.

We are grateful to the patients who participated in this study. We thank all of the investigators who included in this study: Drs Y Aggadi, E Boucher, C Brezault, G Capodano, P Cassan, B Chauffert, P Deguiral, M Ducreux, V Guerrin Meyer, J L Jouve, H Lacroix, J. Lafon, J L Legoux, A Lepoutre, C Lombard Bohas, D Luet, F Mayer, S Nasca, A Pariente, M Picon-Coste, and $\mathrm{M}$ Ychou. We thank the CRAs of the FFCD: Ms C Choine, Mr H Fattouh, Ms F Guiliani, Ms A Kodjo, Mr N Le Provost, Ms M Moreau, and Ms S Ngassam, and administrative executive of the FFCD Ms C Girault. We thank P Bastable for the help in revising the manuscript. We thank also J P Pignon, M Giovannini, and L Thill who participated in the conception and the design of the study. We are grateful to the FNCLCC and to Assistance Publique - Hôpitaux de Marseille that promoted the trial and to Roche that supplied the Roferon.

\section{References}

Arnold R, Rinke A, Klose KJ, Müller HH, Wied M, Zamzow K, Schmidt C, Schade-Brittinger C, Barth P \& Moll R 2005 Octreotide versus octreotide plus interferon- $\alpha$ in endocrine gastropancreatic tumors: a randomized trial. Clinical Gastroenterology and Hepatology 3 761-771.

Arnold R, Müller H, Schade-Brittinger C, Rinke A, Klose K, Barth P, Wied M, Mayer C, Aminossadati B \& PROMID Study Group 2009 Placebo-controlled, double-blind, prospective, randomized study of the effect of octreotide LAR in the control of tumor growth in patients with metastatic neuroendocrine midgut tumors: a report from the PROMID study group. Proceedings of the American Society of Clinical Oncology 274508.

Conseil Scientifique de la FFCD 2003 What can be done for patients with cancer of the digestive tract in 2003? Guidelines of the Francophone Federation of Digestive Tract Cancerology Gastroenterologie Clinique et Biologique 27 43-58.

Detjen KM, Welzel M, Farwig K, Brembeck FH, Kaiser A, Riecken EO, Wiedenmann B \& Rosewicz S 2000 Molecular mechanism of interferon $\alpha$-mediated growth inhibition in human neuroendocrine tumor cells. Gastroenterology 118 735-748.

Dirix LY, Vermeulen PB, Fierens H, De Schepper B, Corthouts B \& Van Oosterom AT 1991 Long-term results 
of continuous treatment with recombinant interferon- $\alpha$ in patients with metastatic carcinoid tumors - an antiangiogenic effect? Anticancer Drugs 7 175-181.

Engstrom PF, Lavin PT, Moertel CG, Folsch E \& Douglass HO Jr 1984 Streptozocin plus fluorouracil versus doxorubicin therapy for metastatic carcinoid tumor. Journal of Clinical Oncology 2 1255-1259.

Eriksson B, Kloppel G, Krenning E, Ahlman H, Plöckinger U, Wiedenmann B, Arnold R, Auernhammer C, Körner M, Rindi G et al. 2008 Consensus Guidelines for the management of patients with digestive neuroendocrine tumors - well differentiated jejunal-ileal tumor/ carcinoma. Neuroendocrinology 87 8-19.

Faiss S, Pape UF, Böhming M, Dorffel Y, Mansmann U, Golder W, Riecken EO, Wiedenmann B \& International Lanreotide and Interferon Alfa Study Group 2003 Prospective, randomized, multicenter trial on the antiproliferative effect of lanreotide, interferon $\alpha$ and their combination for therapy of metastatic neuroendocrine gastropancreatic tumors - the International Lanreotide and Interferon Alfa Study Group. Journal of Clinical Oncology 21 2689-2696.

Gonzalez MA, Biswas S, Clifton L \& Corrie PG 2003 Treatment of neuroendocrine tumours with infusional 5-fluorouracil, folinic acid and streptozocin. British Journal of Cancer 89 455-456.

Haller DG, Schutt A, Dayal Y, Ryan L \& Lipsitz S 1990 Chemotherapy for metastatic carcinoid tumors: an ECOG phase II-III trial. Proceedings of the American Society of Clinical Oncology 9102.

Kolby L, Persson G, Franzen S \& Ahrén B 2003 Randomized clinical trial of the effect of interferon $\alpha$ on survival in patients with disseminated midgut carcinoid tumours. British Journal of Surgery 90 687-693.

Lepage C, Bouvier AM, Phelip JM, Hatem C, Vernet C \& Faivre J 2004 Incidence and management of malignant digestive endocrine tumours in a well defined French population. Gut 53 549-553.

Moertel CG \& Hanley JA 1979 Combination chemotherapy trials in metastatic carcinoid tumor and the malignant carcinoid syndrome. Cancer Clinical Trials $\mathbf{2}$ 327-334.

Moreno A, Akcakanat A, Munsell MF, Soni A, Yao JC \& Meric-Bernstam F 2008 Antitumor activity of rapamycin and octreotide as single agents or in combination neuroendocrine tumors. Endocrine-Related Cancer 15 257-266.
Nave H, Mossinger E, Feist H, Lang H \& Raab H 2001 Surgery as primary treatment in patients with liver metastases from carcinoid tumors: a retrospective, unicentric, study over 13 years. Surgery 129 $170-175$.

Oberg K 1992 The action of interferon $\alpha$ on human carcinoid tumours. Seminars in Cancer Biology 3 35-41.

Oberg K 2000 Interferon in the management of neuroendocrine GEP-tumors: a review. Digestion 62 92-97.

Oberg K \& Eriksson B 1991 The role of interferons in the management of carcinoid tumours. British Journal of Haematology 79 74-77.

Oberg K, Funa K \& Alm G 1983 Effects of leukocyte interferon on clinical symptoms and hormone levels in patients with mid-gut carcinoid tumors and carcinoid syndrome. New England Journal of Medicine 309 129-133.

Oberg K, Norheim I \& Alm G 1989 Treatment of malignant carcinoid tumors: a randomized controlled study of streptozotocin plus 5-FU and human leukocyte interferon. European Journal of Cancer and Clinical Oncology 25 1475-1479.

Pavel ME, Baum U, Hahn EG, Schuppan D \& Lohmann T 2006 Efficacy and tolerability of pegylated IFN- $\alpha$ in patients with neuroendocrine gastroenteropancreatic carcinomas. Journal of Interferon \& Cytokine Research $\mathbf{2 6}$ $8-13$.

Stuart K, Levy DE, Anderson T, Axiotis CA, Dutcher JP, Eisenberg A, Erban JK, Benson AB III \& Eastern Cooperative Oncology Group 2004 Phase II study of interferon gamma in malignant carcinoid tumors (E9292): a trial of the Eastern Cooperative Oncology Group. Investigational New Drugs 22 75-81.

Sun W, Lipsitz S, Catalano P, Mailliard JA, Haller DG \& Eastern Cooperative Oncology Group 2005 Phase II/III study of doxorubicin with fluorouracil compared with streptozocin with fluorouracil or dacarbazine in the treatment of advanced carcinoid tumors: Eastern Cooperative Oncology Group Study E1281. Journal of Clinical Oncology 23 4897-4904.

Yao JC, Phan A, Hoff PM, Chen HX, Charnsangavej C, Yeung SC, Hess K, Ng C, Abbruzzese JL \& Ajani JA 2008 Targeting vascular endothelial growth factor in advanced carcinoid tumor: a random assignement phase II study of depot octreotide with bevacizumab and pegylated interferon $\alpha$-2b. Journal of Clinical Oncology 26 $1316-1323$. 